\title{
A BOUNDARY VALUE PROBLEM FOR COLD PLASMA DYNAMICS
}

\section{THOMAS H. OTWAY}

Received 30 April 2002 and in revised form 18 July 2002

A weak Guderley-Morawetz problem is formulated for a mixed elliptichyperbolic system that arises in models of wave propagation in cold plasma. Weak solutions are shown to exist in a weighted Hilbert space. This result extends the work of Yamamoto (1994).

\section{Introduction}

A characteristic feature of wave propagation in cold plasma is the possibility that a hybrid resonance surface, along which the linearized equation for the scalar potential changes from elliptic to hyperbolic type, may be tangent to a flux surface. This property can be represented in two dimensions by setting the hybrid resonance curve tangent to the line $x=0$ at the origin of coordinates. The situation is somewhat different from that found in, for example, linear models of transonic fluid dynamics, see (1.6). In that case the sonic line is everywhere normal to the line $x=0$.

A model for such a resonance curve is the equation

$$
x=\sigma(y),
$$

where $\sigma(y)$ is a continuously differentiable function of its argument satisfying

$$
\sigma(0)=\sigma^{\prime}(0)=0
$$

In addition, we assume for simplicity that both $\sigma(y)$ and $\sigma^{\prime}(y)$ exceed zero for $y$ exceeding zero. 
18 A boundary value problem for cold plasma dynamics

This leads to consider mixed elliptic-hyperbolic systems having the form

$$
L u=f,
$$

where

$$
\begin{gathered}
u=\left(u_{1}(x, y), u_{2}(x, y)\right), \quad f=\left(f_{1}(x, y), f_{2}(x, y)\right), \\
(x, y) \in \Omega \subset \mathbb{R} \times\left(\mathbb{R} / \mathbb{R}^{-}\right), \\
(L u)_{1}=[x-\sigma(y)] u_{1 x}+K u_{1}+u_{2 y} \\
(L u)_{2}=u_{1 y}-u_{2 x}
\end{gathered}
$$

with data

$$
u_{1} \frac{d x}{d s}+u_{2} \frac{d y}{d s}=0
$$

given on a portion of the boundary of $\Omega$. Here $K$ is a constant in $[0,1]$ and $d s$ denotes the line element on $\partial \Omega$. The system is elliptic for $x>\sigma(y)$ and hyperbolic for $x<\sigma(y)$. Following [7], we emphasize the analogy to fluid dynamics by calling the curve $x=\sigma(y)$ the sonic curve.

In the cold plasma literature, (1.3) and (1.5) tend to appear in scalarvalued special cases. In all these cases $\sigma(y)$ is proportional to $y^{2}$, but this specific restriction is not imposed by the physics; concerning the physical model, see [11, 12]. If $u_{1}=\psi_{x}, u_{2}=\psi_{y}, \sigma(y)=y^{2}$, and $f=(0,0)$, the system reduces to a scalar equation introduced in [7, Section 3$]$. In the context of this equation, condition (1.5) corresponds to imposing constant boundary conditions on the scalar solution $\psi(x, y)$. A uniqueness theorem was proven in [7] for $K=1 / 2$, in order to show the existence of a domain on which the classical Dirichlet problem is ill-posed for the equation. Numerical arguments for a complex perturbation were also introduced. A similar equation, $\sigma(y) \propto y^{2}, u_{1}=\psi_{x}, u_{2}=-\psi_{y}, K=1, f=(0,0)$, appeared earlier in the physics literature, also in the context of wave propagation in cold plasma [10]. In this case certain exact solutions were constructed. Finally, system (1.3) and (1.5) in the case $\sigma(y)=y^{2}$ was studied in an interesting Ph.D. dissertation [13] on the existence of weak solutions possessing Dirichlet data on a certain small domain near the origin of $\mathbb{R}^{2}$.

These equations share with the Tricomi equation,

$$
y \psi_{x x}+\psi_{y y}=0
$$

a multiplicity of possible approaches to formulating boundary value problems. From a mathematical point of view, the Dirichlet problem, in 
which data are assigned on the entire boundary, is the "wrong problem" to solve for equations of mixed type, as this problem tends to become over-determined in the hyperbolic region. Well-posed problems for elliptic-hyperbolic equations generally include a characteristic gap on which data have not been prescribed. In addition to the example of an over-determined Dirichlet problem for the scalar equation considered in [7], there are analogous examples for Tricomi-like equations [4].

However, physical applications of (1.6) to transonic fluid dynamics, and of scalar forms of (1.3) to wave propagation in cold plasma, suggest that it should be possible to prescribe data over the entire boundary. This contradiction suggests that classical solutions will have little application to such physical problems. In terms of weak solutions $u$ to system (1.3), the Dirichlet problem requires solutions to lie in an appropriate Hilbert space $\widetilde{H_{1}}$ and satisfy

$$
-\left(u, L^{*} \varphi\right)_{L^{2}}=(f, \varphi)_{L^{2}}
$$

for all $\varphi=\left(\varphi_{1}, \varphi_{2}\right) \in \widetilde{H}_{2}$, where $\widetilde{H}_{2}$ is another appropriate Hilbert space and where $\varphi_{1}=0$ on the entire boundary of the domain (cf. [6, equations (6)-(8)], for the Tricomi case). This problem may or may not be wellposed. A different approach is to require that the component $\varphi_{1}$ of the test function $\varphi$ vanishes only on the noncharacteristic part of the boundary, and that $\varphi$ satisfies condition (1.5) on characteristics. In the early literature (see, e.g., [5, Section 4]) an elliptic-hyperbolic problem having Dirichlet data given on the entire boundary is called the closed, or full Dirichlet problem to distinguish it from the mathematically natural case of Dirichlet data given only on part of the boundary. Following [2], we prefer instead to distinguish the problem in which data are given on the complement of a characteristic gap by calling such a problem GuderleyMorawetz, reserving the term Dirichlet problem for Dirichlet data given on the entire boundary as in $[6,9]$.

The existence of weak solutions to a Guderley-Morawetz problem is proven for a Tricomi-like system in [3]. The estimates in [13, Section 2.5] can be extended to imply the existence of weak solutions to a GuderleyMorawetz problem for (1.3) and (1.5) on a relatively large and general domain. This is the content of Theorems 3.1 and 3.2. The arguments in [13] assume that the weak Dirichlet problem and the weak GuderleyMorawetz problems are identical. See Remark 2.2. It is claimed in [13], on the basis of Guderley-Morawetz estimates modeled on [3], that weak solutions of (1.3) exist for Dirichlet data prescribed on the entire boundary. We make no such claim for the generalization of those estimates given here. However, the techniques used to prove the existence of weak solutions to a Guderley-Morawetz problem for (1.3) and (1.5) will yield 
a uniqueness theorem for strong solutions to the Dirichlet problem for this system, on a more restricted domain, almost for free (see Section 3.3; also see [13, Theorem 1, Section 2.7]). Moreover, it is possible to derive the existence of weak solutions to the Dirichlet problem for (1.6) by considering a sequence of Guderley-Morawetz problems in which the characteristic gap is "marched" to a singular point on the sonic curve; see $[1,5,9]$. It is a reasonable conjecture that this method can be modified to apply to systems such as system (1.3) and (1.5) on an appropriate domain, but this is not attempted here.

Equations (1.3) cannot be mapped into a system of the form studied in [3] on any domain that includes the origin. On the one hand, relatively little is known about such elliptic-hyperbolic systems which do not directly generalize the Tricomi equation. On the other hand, the method of proof adopted here is by now quite standard. It is required to find a Hilbert space $U$, a domain $\Omega$, and a multiplier $M$ under which weak solutions can be shown to exist, without unreasonable restrictions on generality, by a uniqueness-plus-projection argument using the $a b c$ method and the Riesz representation theorem. Because this system comes from a physical model, we additionally hope that our conditions on $U$ and $\Omega$ will be physically reasonable. For instance, physical/numerical arguments for special cases suggest that a singularity should be permitted at the origin [7]. This influences the weighting of the Hilbert space $U$, as does the existence of particular physical solutions (Section 2.2.2).

We note that every nondegenerate conic section is equivalent under the projective group to the unit circle. In that sense, system (1.3) and (1.5) with the choice $\sigma(y)=y^{2}$ is gauge equivalent to a system on the extended projective disc studied in [8] by similar methods. In that system, the elliptic part of the domain has a geometric interpretation as hyperbolic points in projective space and the hyperbolic part, as ideal points. As it is not clear that projective invariance has any physical meaning in the context of cold plasma dynamics, this analogy will not be pursued.

\section{Formulation of the boundary value problem}

\subsection{Domain}

In proving weak existence for the Guderley-Morawetz problem we assume that the domain $\Omega$, having piecewise continuous boundary, is enclosed by the arbitrarily large but finite rectangle

$$
R=\{(x, y) \mid-\infty<p \leq x<\ell, 0 \leq y \leq q<\infty\},
$$

where $\ell, p, q$ are fixed but arbitrary real constants. We assume that $R$ has been chosen so that the distance along the $x$-axis from $\sup _{\Omega} x$ to $\ell$ is 
an arbitrary but fixed positive number $\delta$. Because we are assuming the existence of an elliptic region for (1.3), we take $\ell>0$.

The elliptic region of $\Omega$ consists of the region of the first quadrant bounded by the sonic curve $x=\sigma(y)$ and a smooth curve $C_{1}$ emerging from the origin, along which

$$
\frac{d y}{d x} \geq 0
$$

with equality only at the origin, and

$$
a(y) \frac{d y}{d x}+b(y)<0
$$

for specified functions $b(y) \leq 0$ and $a(y) \geq 0$. We assume that $C_{1}$ intersects the sonic curve at a point $\left(\sigma\left(y_{0}\right), y_{0}\right) \in \Omega$, where $y_{0}>0$. For example, if $a, b$, and $\sigma$ are defined as in Theorem 3.2, then the family of curves given by $y=\varepsilon x^{m}$ for $m>1 / 2$ and $x \geq 0$ satisfies condition (2.3) for

$$
\varepsilon \leq \sqrt{\frac{\ell^{1-2 m}}{K m}}
$$

whenever $K>0$. (Condition (2.3) is automatically satisfied for such $a, b$, and $\sigma$ if $K=0$.) If we further specify $m \leq 1 / K$, then we guarantee that $\sigma\left(y_{0}\right) \leq \ell-\delta$ provided we choose

$$
\varepsilon \geq \sqrt{(\ell-\delta)^{1-2 m}}
$$

The hyperbolic region is bounded by a piecewise smooth curve $\Gamma \cup C_{2}$, where $\Gamma$ is a characteristic curve

$$
\frac{d x}{d y}=-\sqrt{\sigma(y)-x}
$$

emerging from the sonic curve at $\left(\sigma\left(y_{0}\right), y_{0}\right) ; C_{2}$ is a piecewise continuous curve intersecting the characteristic $\Gamma$ at a single point on the left endpoint of $C_{2}$ and intersecting $C_{1}$ at the origin on the right endpoint of $C_{2}$. We assume that $d y \leq 0$ and $d x \geq 0$ on $C_{2}$. We orient the boundary in the counterclockwise direction.

The relation of this domain to the domain considered in [13] is discussed at the end of Section 3.3. 
22 A boundary value problem for cold plasma dynamics

\subsection{Function spaces}

\subsubsection{Weak solutions}

Denote by $U$ the Hilbert space consisting of all pairs of measurable functions $\left(u_{1}, u_{2}\right)$ such that

$$
\|u\|_{*}=\left[\iint_{\Omega} \sigma^{\prime}(y)\left(u_{1}^{2}+u_{2}^{2}\right) d x d y\right]^{1 / 2}
$$

is finite. Here

$$
(u, w)_{*}=\iint_{\Omega} \sigma^{\prime}(y)\left(u_{1} w_{1}+u_{2} w_{2}\right) d x d y
$$

Denote by $W$ the linear space of continuously differentiable functions $\left(w_{1}, w_{2}\right)$ vanishing at the origin of $\mathbb{R}^{2}$ and satisfying

$$
w_{1} d x+w_{2} d y=0
$$

on the characteristic $\Gamma, w_{1}=0$ on $\partial \Omega / \Gamma$, and

$$
\left\{\iint_{\Omega} \frac{1}{\sigma^{\prime}(y)}\left[\left(L^{*} w\right)_{1}^{2}+\left(L^{*} w\right)_{2}^{2}\right] d x d y\right\}^{1 / 2}<\infty
$$

where

$$
\begin{aligned}
& \left(L^{*} w\right)_{1}=[x-\sigma(y)] w_{1 x}+(1-K) w_{1}+w_{2 y} \\
& \left(L^{*} w\right)_{2}=w_{1 y}-w_{2 x} .
\end{aligned}
$$

We define a weak solution to (1.3) under the boundary condition (1.5) to be any $u \in U$ such that for all $w \in W$,

$$
(w, f)=-\left(L^{*} w, u\right)
$$

under the $L^{2}$ inner product (,).

Denote by $H$ the Hilbert space of measurable functions $\left(h_{1}, h_{2}\right)$ for which the norm

$$
\|h\|^{*}=\left[\iint_{\Omega} \frac{1}{\sigma^{\prime}(y)}\left(h_{1}^{2}+h_{2}^{2}\right) d x d y\right]^{1 / 2}
$$


is finite. The inner product on $H$ is given by

$$
(h, g)^{*}=\iint_{\Omega} \frac{1}{\sigma^{\prime}(y)}\left(h_{1} g_{1}+h_{2} g_{2}\right) d x d y .
$$

The prescribed data $f$ will be assumed to lie in the space $H$.

\subsubsection{Similarity solutions}

Analysis of scalar special cases of system (1.3) with $\sigma(y)=y^{2}$ suggests the presence of a singularity at the point $x=y=0$. See, for example, [7], where this is discussed in detail. This would suggest a radial weight for the energy functional. Quadratic radial weights are applied in [13]. However, the space $U$ constructed here also arises naturally in connection with (1.3).

As a simple example, consider similarity solutions for the case $\sigma(y)=$ $y^{2}$ having the form $u_{1}=\psi_{x}, u_{2}=\psi_{y}$, and

$$
\psi(x, y)=x^{v} F\left(\frac{y^{2}}{x}\right),
$$

where $v$ is a parameter and $F$ satisfies the hypergeometric equation

$$
\begin{gathered}
(1-\mu)\left[v(v-1) F(\mu)-2(v-1) \mu F^{\prime}(\mu)+\mu^{2} F^{\prime \prime}(\mu)\right] \\
+\left[2 F^{\prime}(\mu)+4 \mu F^{\prime \prime}(\mu)\right]=0
\end{gathered}
$$

for

$$
\mu=\frac{y^{2}}{x} .
$$

Properties of such solutions for complex values of $v$ are studied in [7]. We consider here the case of real-valued $v$, as in $[10,11]$. It has been observed [11] that if $|x|$ is sufficiently small, then $F \sim \mu^{\nu}$ or $F \sim \mu^{\nu-1}$. Taking $F \sim \mu^{v}$ for $v=1 / 4$, we find that

$$
\psi(x, y)=x^{1 / 4} F\left(\frac{y^{2}}{x}\right) \sim y^{1 / 2} .
$$

If $u$ lies in the function space $U$, then $\psi$ has weighted Dirichlet norm

$$
E_{U}(\psi)=\|u\|_{*}^{2}=2 \int_{\Omega} y\left(\psi_{x}^{2}+\psi_{y}^{2}\right) d x d y \sim \frac{\operatorname{vol}(\Omega)}{2} .
$$

In fact, $E_{U}(\psi)$ is finite on $\Omega$ for all $v \geq 1 / 4$. If we include solutions of the form $F \sim \mu^{\nu-1}$, then $E_{U}(\psi)$ would be finite on $\Omega$ for all $v \geq 5 / 4$. 
24 A boundary value problem for cold plasma dynamics

\subsection{The weak problem is well-posed}

Proposition 2.1. Any continuously differentiable weak solution of the Guderley-Morawetz problem for (1.3) and (1.5) on $\Omega$, as defined by (2.12), is a classical solution.

Proof. We refer to the domain as $\Omega$, but the argument also holds without alteration on much more general domains. For $u \in U$ and $w \in W$, integration by parts yields

$$
\begin{aligned}
\left(u, L^{*} w\right)= & \iint_{\Omega} u_{1}\left\{[x-\sigma(y)] w_{1 x}+(1-K) w_{1}+w_{2 y}\right\} d x d y \\
& +\iint_{\Omega} u_{2}\left(w_{1 y}-w_{2 x}\right) d x d y \\
= & -\iint_{\Omega}\left\{[x-\sigma(y)] u_{1 x}+K u_{1}+u_{2 y}\right\} w_{1} d x d y \\
& -\iint_{\Omega}\left(u_{1 y}-u_{2 x}\right) w_{2} d x d y-\int_{\partial \Omega}\left(w_{1} u_{2}+w_{2} u_{1}\right) d x \\
& +\int_{\partial \Omega}\left\{[x-\sigma(y)] w_{1} u_{1}-w_{2} u_{2}\right\} d y
\end{aligned}
$$

On $\partial \Omega / \Gamma, w_{1}=0$, implying that

$$
\left(u, L^{*} w\right)_{\mid \partial \Omega / \Gamma}=-\int_{\partial \Omega / \Gamma} w_{2}\left(u_{1} d x+u_{2} d y\right)
$$

Equations (2.6) and (2.9) hold on $\Gamma$, implying that

$$
\begin{aligned}
(u, L w)_{\mid \Gamma} & =\int_{\Gamma}-\left(w_{1} u_{2}+w_{2} u_{1}\right) d x+\left\{[x-\sigma(y)] w_{1} u_{1}-w_{2} u_{2}\right\} d y \\
& =\int_{\Gamma} u_{1}\left\{[x-\sigma(y)] w_{1}-w_{2} \frac{d x}{d y}\right\} d y-u_{2}\left(w_{1} d x+w_{2} d y\right) \\
& =\int_{\Gamma}\left[x-\sigma(y)+\left(\frac{d x}{d y}\right)^{2}\right] w_{1} u_{1} d y=0
\end{aligned}
$$

Substituting (2.21) and (2.22) into (2.20) and using (2.12), we obtain

$$
-(w, f)=\left(u, L^{*} w\right)=-(L u, w)-\int_{\partial \Omega / \Gamma} w_{2}\left(u_{1} d x+u_{2} d y\right)
$$


Because this identity must hold for every $w \in W$, we conclude that the quantity $u_{1} d x+u_{2} d y$ must equal zero almost everywhere on $\partial \Omega / \Gamma$. Applying the hypothesis that $u$ is continuously differentiable, we complete the proof of Proposition 2.1.

Remark 2.2. The value of the 1 -form $u_{1} d x+u_{2} d y$ on the characteristic $\Gamma$ is left undetermined by a definition of weak solution based on (2.12), so this argument will not establish the well-posedness of the weak Dirichlet problem for (1.3) and (1.5) on $\Omega$ (unless we change the boundary conditions on $w$ to $w_{1}=0$ on $\partial \Omega$ ). However, classical solutions $u$ of either the Dirichlet problem or the Guderley-Morawetz problem satisfy (2.12). This ambiguity seems to be the basis for the attempt in [13] to identify the weak forms of the two problems; (cf. [13, Section 2.3]).

\section{Results}

Theorem 3.1. Let $K \in[0,1 / 2]$. Let the functions $a(y)$ and $b(y)$ in condition (2.3) be given by

$$
\begin{aligned}
& a(y)=K\left[y+\ell^{-1} \int_{0}^{y} \sigma(t) d t\right], \\
& b(y)=-\left[1+\frac{\sigma(y)}{\ell}\right] .
\end{aligned}
$$

For every $f \in H$, there exists on $\Omega$ a weak solution to system (1.3) with the boundary condition (1.5) given on $\partial \Omega / \Gamma$.

THeOREM 3.2. The conclusion of Theorem 3.1 extends to the case $K \in[0,1]$ if the definitions of $a(y)$ and $b(y)$ are replaced by

$$
\begin{aligned}
& a(y)=K y, \\
& b(y)=-\left[1+\frac{\sigma(y)}{2 \ell}\right],
\end{aligned}
$$

and specify $\sigma(y)=y^{2}$.

The proofs of Theorems 3.1 and 3.2 modify the argument in [3]. In addition, we adapt a number of choices made in [13], which is also based on [3]. The results follow from an a priori estimate.

Lemma 3.3. Under the hypotheses of either Theorem 3.1 or Theorem 3.2, there exists $k>0$ such that for all $w \in W$,

$$
k\|w\|_{*} \leq\left\|L^{*} w\right\|^{*} .
$$


26 A boundary value problem for cold plasma dynamics

\subsection{Proof of Lemma 3.3}

We prove the lemma by the $a b c$ method. Let

$$
M=\left[\begin{array}{ll}
a & b \\
c & d
\end{array}\right],
$$

where $a$ and $b$ are given by the hypotheses of the Theorems 3.1 and 3.2; $c$ and $d$ will be chosen. Then

$$
\begin{aligned}
I= & \left(L^{*} w, M w\right) \\
= & \iint_{\Omega}\left\{[x-\sigma(y)] w_{1 x}+(1-K) w_{1}+w_{2 y}\right\}\left(a w_{1}+b w_{2}\right) d x d y \\
& +\iint_{\Omega}\left(w_{1 y}-w_{2 x}\right)\left(c w_{1}+d w_{2}\right) d x d y .
\end{aligned}
$$

Notice that $a$ and $b$ are defined so that $a_{x}=b_{x}=0$. The idea of the proof is to estimate $I$ from above and below. Proceeding as in [13, Section 2.4], we write

$$
\begin{aligned}
a[x-\sigma(y)] w_{1} w_{1 x} & =\frac{1}{2}\left(\left\{a[x-\sigma(y)] w_{1}^{2}\right\}_{x}-a w_{1}^{2}\right) \\
b w_{2}[x-\sigma(y)] w_{1 x} & =\left\{b[x-\sigma(y)] w_{1} w_{2}\right\}_{x}-b w_{1} w_{2}-b[x-\sigma(y)] w_{1} w_{2 x} \\
a w_{1} w_{2 y} & =\left(a w_{1} w_{2}\right)_{y}-\frac{1}{2}\left(a w_{2}^{2}\right)_{x}-a_{y} w_{1} w_{2}+a w_{2} w_{2 x}-a w_{1 y} w_{2} \\
b w_{2} w_{2 y} & =\frac{1}{2}\left[\left(b w_{2}^{2}\right)_{y}-b_{y} w_{2}^{2}\right] \\
c w_{1 y} w_{1} & =\frac{1}{2}\left[\left(c w_{1}^{2}\right)_{y}-c_{y} w_{1}^{2}\right]
\end{aligned}
$$

with the choices $d=a$ and

$$
c=-b[x-\sigma(y)]
$$

Taking into account cancellations, we can write $I=I_{1}+I_{2}$, where $I_{2}$ is a line integral and

$$
I_{1}=\iint_{\Omega}\left(\alpha w_{1}^{2}+2 \beta w_{1} w_{2}+\gamma w_{2}^{2}\right) d x d y
$$


for

$$
\begin{aligned}
& \alpha=\frac{1}{2}\left\{b_{y}[x-\sigma(y)]-b(y) \sigma^{\prime}(y)\right\}+\left(\frac{1}{2}-K\right) a(y) \\
& \beta=-\frac{1}{2}\left[a_{y}+K b(y)\right] \\
& \gamma=-\frac{1}{2} b_{y} .
\end{aligned}
$$

Case 1. Under the hypothesis on $K$ in Theorem 3.1, the coefficient of $a(y)$ in $\alpha$ is nonnegative, and we can write

$$
\begin{aligned}
\alpha & =\frac{\sigma^{\prime}(y)}{2 \ell}[2 \sigma(y)+\ell-x]+K\left(\frac{1}{2}-K\right)\left[y+\ell^{-1} \int_{0}^{y} \sigma(t) d t\right] \\
& \geq \frac{\sigma^{\prime}(y)}{2 \ell}[2 \sigma(y)+\ell-x] \geq \frac{\delta \sigma^{\prime}(y)}{2 \ell} \\
\beta & =0 \\
\gamma & =\frac{\sigma^{\prime}(y)}{2 \ell} .
\end{aligned}
$$

Thus in this case we have

$$
I_{1} \geq \iint_{\Omega}\left(\alpha w_{1}^{2}+\gamma w_{2}^{2}\right) d x d y \geq \frac{x}{2 \ell} \iint_{\Omega} \sigma^{\prime}(y)\left(w_{1}^{2}+w_{2}^{2}\right) d x d y
$$

where $x=\min \{\delta, 1\}$.

Case 2. Under the hypotheses of Theorem 3.2, we have

$$
\begin{aligned}
\alpha & =\frac{y}{2 \ell}\left(2 y^{2}+2 \ell-x\right)+\left(\frac{1}{2}-K\right) K y \geq \frac{y}{2 \ell}\left(2 y^{2}+2 \ell-x\right)-\frac{y}{2} \\
& =\frac{y}{2 \ell}\left(2 y^{2}+\ell-x\right) \geq \frac{\delta y}{2 \ell}, \\
\beta & =\frac{K y^{2}}{4 \ell}, \\
\gamma & =\frac{y}{2 \ell} .
\end{aligned}
$$


28 A boundary value problem for cold plasma dynamics

Notice that

$$
\begin{aligned}
\alpha \gamma-\beta^{2} & \geq\left(\frac{y}{2 \ell}\right)^{2}\left(2 y^{2}+\ell-x\right)-\left(\frac{K y^{2}}{4 \ell}\right)^{2} \\
& \geq\left(\frac{y}{2 \ell}\right)^{2}\left[\ell-x+\frac{7}{4} y^{2}\right] \geq \delta\left(\frac{y}{2 \ell}\right)^{2} .
\end{aligned}
$$

Cauchy's inequality implies that

$$
2 \beta w_{1} w_{2} \geq-2|\beta|\left|w_{1}\right|\left|w_{2}\right|>-2 \sqrt{\alpha}\left|w_{1}\right| \sqrt{\gamma}\left|w_{2}\right| \geq-\alpha w_{1}^{2}-\gamma w_{2}^{2}
$$

in $\Omega /\{y=0\}$. This already implies that the $W$-norm of $w$ is positive inside the upper half-plane. It remains, however, to derive an explicit lower bound on the coefficient of $y\left(w_{1}^{2}+w_{2}^{2}\right)$.

We claim that there is a constant $\varepsilon \in(0,1)$, depending only on $R$, for which

$$
0 \leq \alpha \gamma-\delta\left(\frac{y}{2 \ell}\right)^{2} \leq \varepsilon \alpha \gamma
$$

To establish this claim, note that the left-hand inequality in (3.15) is obvious from (3.13), and the right-hand inequality will be satisfied provided

$$
\alpha \gamma(1-\varepsilon) \leq \delta\left(\frac{y}{2 \ell}\right)^{2}
$$

Assuming without loss of generality that $y$ exceeds zero (the inequality is true trivially otherwise), our criterion becomes

$$
2\left\{y^{2}+\ell\left[1+\left(\frac{1}{2}-K\right) K\right]\right\}-x \leq \frac{\delta}{1-\varepsilon} .
$$

Replace the quantity on the left by its largest possible value, given that $(1 / 2-K) K \leq 1 / 16$. Our requirement becomes that $\varepsilon$ be chosen sufficiently close to 1 so that

$$
2 q^{2}+\frac{17 \ell}{8}-p \leq \frac{\delta}{1-\varepsilon}
$$

or

$$
1-\frac{\delta}{2 q^{2}+17 \ell / 8-p} \leq \varepsilon
$$

The quantity on the left exceeds zero, as $\ell-p$ exceeds $\delta$. 
Now (3.13) and (3.15) imply that inequality (3.14) can be improved to read

$$
\begin{aligned}
2 \beta w_{1} w_{2} & \geq-2|\beta|\left|w_{1}\right|\left|w_{2}\right| \geq-2 \sqrt{\alpha \gamma-\delta\left(\frac{y}{2 \ell}\right)^{2}}\left|w_{1}\right|\left|w_{2}\right| \\
& \geq-2 \sqrt{\varepsilon \alpha \gamma}\left|w_{1}\right|\left|w_{2}\right| \geq-\sqrt{\varepsilon} \alpha w_{1}^{2}-\sqrt{\varepsilon} \gamma w_{2}^{2} .
\end{aligned}
$$

Thus, in this case

$$
\begin{aligned}
I_{1} & \geq(1-\sqrt{\varepsilon}) \iint_{\Omega}\left(\alpha w_{1}^{2}+\gamma w_{2}^{2}\right) d x d y \\
& \geq \frac{(1-\sqrt{\varepsilon})}{2 \ell} \iint_{\Omega} y\left(\delta w_{1}^{2}+w_{2}^{2}\right) d x d y \\
& \geq \frac{x(1-\sqrt{\varepsilon})}{2 \ell} \iint_{\Omega} y\left(w_{1}^{2}+w_{2}^{2}\right) d x d y .
\end{aligned}
$$

The remainder of the proof is identical for either set of hypotheses. The boundary terms resulting from applying Green's theorem on $\Omega$ are given by

$$
\begin{aligned}
I_{2}= & -\int_{\partial \Omega}\left(\frac{b}{2}\left\{w_{2}^{2}-[x-\sigma(y)] w_{1}^{2}\right\}+a w_{1} w_{2}\right) d x \\
& +\int_{\partial \Omega}\left(\frac{a}{2}\left\{[x-\sigma(y)] w_{1}^{2}-w_{2}^{2}\right\}+b[x-\sigma(y)] w_{1} w_{2}\right) d y
\end{aligned}
$$

On $C_{2}, w_{1}=0, d x \geq 0$ and $d y \leq 0$, so the signs of $a$ and $b$ imply that

$$
I_{2 \mid C_{2}}=-\frac{1}{2} \int_{C_{2}} a w_{2}^{2} d y+b w_{2}^{2} d x \geq 0 .
$$

On the characteristic $\Gamma,(2.6)$ and (2.9) imply that

$$
w_{2}^{2}+[x-\sigma(y)] w_{1}^{2}=w_{1}^{2}[\sigma(y)-x]+[x-\sigma(y)] w_{1}^{2}=0
$$

We have

$$
I_{2 \mid \Gamma}=I_{21}+I_{22}
$$


30 A boundary value problem for cold plasma dynamics

where

$$
\begin{aligned}
I_{21} & =-\int_{\Gamma} a w_{1} w_{2} d x+\frac{1}{2} \int_{\Gamma} a\left\{[x-\sigma(y)] w_{1}^{2}-w_{2}^{2}\right\} d y \\
& =\int_{\Gamma} \frac{a}{2}\left\{w_{2}^{2}+[x-\sigma(y)] w_{1}^{2}\right\} d y=0, \\
I_{22} & =-\int_{\Gamma} \frac{b}{2}\left\{w_{2}^{2}-[x-\sigma(y)] w_{1}^{2}\right\} d x+\int_{\Gamma} b[x-\sigma(y)] w_{1} w_{2} d y \\
& =-\int_{\Gamma} \frac{b}{2}\left\{w_{2}^{2}+[x-\sigma(y)] w_{1}^{2}\right\} d x=0 .
\end{aligned}
$$

On $C_{1}$ inequality (2.3) holds; in addition, $d x \geq 0, d y \geq 0$, and $w_{1}=0$. Writing

$$
\int_{C_{1}} a w_{2}^{2} d y=\int_{C_{1}} a w_{2}^{2} \frac{d y}{d x} d x
$$

we find that

$$
\begin{aligned}
I_{2 \mid C_{1}} & =-\frac{1}{2} \int_{C_{1}} a w_{2}^{2} d y+b w_{2}^{2} d x \\
& =-\frac{1}{2} \int_{C_{1}}\left[a \frac{d y}{d x}+b\right] w_{2}^{2} d x .
\end{aligned}
$$

Inequality (2.3) implies that the integral on the right is nonnegative.

The preceding arguments establish the lower bound of the lemma.

In order to obtain the upper bound for the inequality of Lemma 3.3 we reason in either Case 1 or Case 2 as in [3], writing

$$
I=\lim _{\tau \rightarrow 0}\left(\frac{L^{*} w}{\sqrt{\sigma^{\prime}(y)+\tau}},\left(\sqrt{\sigma^{\prime}(y)+\tau}\right) M w\right) \leq C(M)\left\|L^{*} w\right\|^{*}\|w\|_{*} .
$$

The constant $C(M)$ will be a finite positive number provided the functions $a, b, c, d$ are bounded. The existence of such a bound follows from the finite character of the constants $p, q$, and $\ell$.

We obtain, under the hypotheses of either theorem, the inequality

$$
C^{\prime}(p, q, \ell, \varepsilon, \delta)\|w\|_{*} \leq\left\|L^{*} w\right\|^{*}
$$

for $C^{\prime}>0$. This completes the proof of Lemma 3.3. 


\subsection{Proof of Theorems 3.1 and 3.2}

Both theorems follow from Lemma 3.3 by a standard argument. An inequality similar to (3.29) implies that for all $w \in W$,

$$
|(w, f)| \leq c_{0}\left\|L^{*} w\right\|^{*}\|f\|^{*}
$$

for a constant $c_{0}$ depending only on $\Omega$. For fixed $f \in H$, the functional

$$
G\left(L^{*} w\right) \equiv(w, f)
$$

on $L^{*} w$ can be extended to a bounded linear functional on $H$. The Riesz representation theorem then implies the existence of an element $h=$ $\left(h_{1}, h_{2}\right) \in H$ for which

$$
(w, f)=\left(L^{*} w, h\right)^{*} .
$$

Defining $u=\left(u_{1}, u_{2}\right)$, where

$$
\begin{aligned}
& u_{1}=-\frac{h_{1}}{\sigma^{\prime}(y)}, \\
& u_{2}=-\frac{h_{2}}{\sigma^{\prime}(y)},
\end{aligned}
$$

we find that $u \in U$ and

$$
(w, f)=\left(L^{*} w, h\right)^{*}=-\left(L^{*} w, u\right)
$$

for all $w \in W$, which completes the proof.

\subsection{Remark}

By slightly modifying the proof of Lemma 3.3 it is possible to prove the uniqueness of strong solutions to a Dirichlet problem on a more restricted domain. Replace $\Omega$ by a domain $\Omega^{\prime}$, in which $C_{2}$ is replaced by the piecewise linear curve $\lambda_{1} \cup \lambda_{2}$, where $\lambda_{1}$ is a vertical line segment $x=$ const $<0$, lying in the interior of $R$, bounded above by $\Gamma$ and below by $\lambda_{2}$; such vertical lines correspond to flux surfaces in the cold plasma model; $\lambda_{2}$ is the segment of the $x$-axis bounded on the left by the line segment $\lambda_{1}$ and on the right by the line $x=0$. The curves $C_{1}$ and $\Gamma$ are identically defined on $\Omega$ and $\Omega^{\prime}$. Let condition (2.3) be satisfied for $b(y)$ defined as in Theorem 3.1 and for

$$
a(y)=(1-K)\left[y+\ell^{-1} \int_{0}^{y} \sigma(t) d t\right] .
$$


32 A boundary value problem for cold plasma dynamics

Let $K$ lie in the interval $[1 / 2,1]$. Then for every $f \in H$ there exists at most one strong solution in $U$ to (1.3) on $\Omega^{\prime}$ with the boundary condition (1.5) given on almost all of $\partial \Omega^{\prime}$. This conclusion extends to the case $K \in[0,1]$ if $b(y)$ and $\sigma(y)$ are defined as in Theorem 3.2 and $a(y)=(1-K) y$.

By a strong solution of (1.3) we mean an element $u \in U$ for which there exists a sequence $u^{v} \in U$ such that

$$
\begin{aligned}
\lim _{v \rightarrow \infty}\left\|u^{v}-u\right\|_{*} & =0, \\
\lim _{v \rightarrow \infty}\left\|L u^{v}-f\right\|^{*} & =0 .
\end{aligned}
$$

This strong solution satisfies the boundary condition (1.5) on almost all of $\partial \Omega^{\prime}$ if in addition

$$
\int_{\partial \Omega^{\prime}}\left(u_{1}^{v} d x+u_{2}^{v} d y\right)^{2}(d s)^{-1}=0
$$

where $d s$ is the line element on $\partial \Omega^{\prime}$.

Suppose that we impose the following additional restrictions and modifications on the domain $\Omega^{\prime}$ : the arbitrarily large finite rectangle $R$ in the upper half-plane is replaced by a sufficiently small circle $R_{0}$ in the upper half-plane, tangent to the origin; the line segment $\lambda_{1}$ is chosen to lie sufficiently close to the $y$-axis; the line segment $\lambda_{2}$ is replaced by that segment of $R_{0}$ bounded on the left by $\lambda_{1}$ and on the right by the $y$-axis; the characteristic curve $\Gamma$ is a curve satisfying

$$
\frac{d x}{d y}=-\sqrt{y^{2}-x}
$$

emerging from the parabola $x=y^{2}$ at a point $\left(\tilde{\delta}, \tilde{\delta}^{2}\right)$ sufficiently close to the origin; condition (2.3) is satisfied for $b(y)=-\left(1+y^{2}\right)$ and $a(y)=$ $(1-K) y$.

Then $\Omega^{\prime}$ becomes identical to the domain $D$ considered in $[13$, Chapter 2]. A uniqueness theorem for solutions of (1.3) and (1.5), with $\sigma(y)=$ $y^{2}$, lying in a radially weighted Hilbert space over $D$ is given in [13, Section 2.7].

\section{References}

[1] K. G. Guderley, The Theory of Transonic Flow, Pergamon Press, Oxford, 1962.

[2] D. Lupo and K. R. Payne, A dual variational approach to a class of nonlocal semilinear Tricomi problems, NoDEA Nonlinear Differential Equations Appl. 6 (1999), no. 3, 247-266.

[3] C. S. Morawetz, A weak solution for a system of equations of elliptic-hyperbolic type, Comm. Pure Appl. Math. 11 (1958), 315-331. 
[4] Non-existence of transonic flow past a profile, Comm. Pure Appl. Math. 17 (1964), 357-367.

[5] - Mixed equations and transonic flow, Rend. Mat. Appl. (5) 25 (1966), 482-509.

[6] - The Dirichlet problem for the Tricomi equation, Comm. Pure Appl. Math. 23 (1970), 587-601.

[7] C. S. Morawetz, D. C. Stevens, and H. Weitzner, A numerical experiment on a second-order partial differential equation of mixed type, Comm. Pure Appl. Math. 44 (1991), no. 8-9, 1091-1106.

[8] T. H. Otway, Hodge equations with change of type, Ann. Mat. Pura Appl. 181 (2002), no. 4, 427-436.

[9] K. R. Payne, Boundary geometry and location of singularities for solutions to the Dirichlet problem for Tricomi type equations, Houston J. Math. 23 (1997), no. 4, 709-731.

[10] A. D. Piliya and V. I. Fedorov, Singularities of the field of an electromagnetic wave in a cold anisotropic plasma with two-dimensional inhomogeneity, Soviet Phys. JETP 33 (1971), 210-215.

[11] H. Weitzner, Wave propagation in a plasma based on the cold plasma model, Tech. Report MF-103, Magneto-Fluid Dynamics Division, Courant Institute of Mathematical Sciences, New York University, New York, 1984.

[12] L Lower hybrid waves in the cold plasma model, Comm. Pure Appl. Math. 38 (1985), no. 6, 919-932.

[13] Y. Yamamoto, Existence and uniqueness of a generalized solution for a system of equations of mixed type, Ph.D. thesis, Polytechnic University, New York, 1994.

Thomas H. Otway: Departments of Mathematics and Physics, Yeshiva University, Wilf Campus, 500 West 185th Street, New York, NY 10033, USA

E-mail address: otway@ymail.yu.edu 


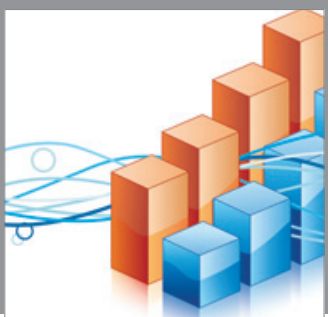

Advances in

Operations Research

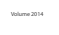

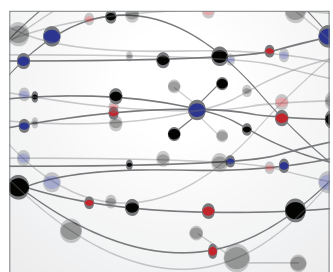

\section{The Scientific} World Journal
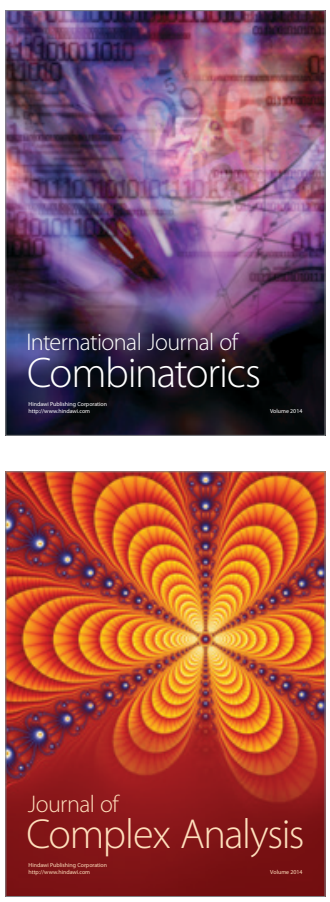

International Journal of

Mathematics and

Mathematical

Sciences
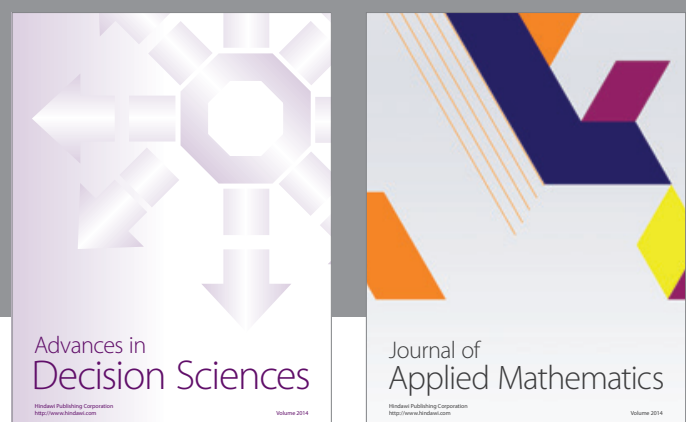

Journal of

Applied Mathematics
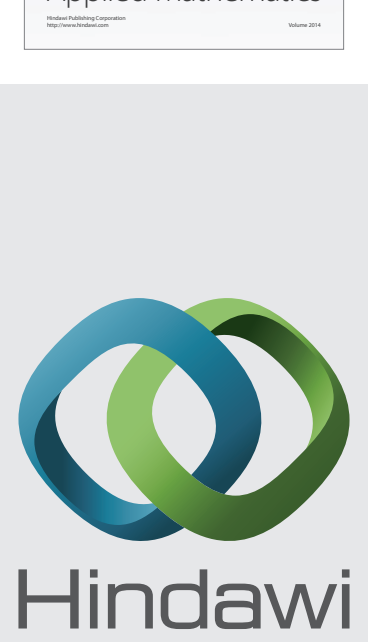

Submit your manuscripts at http://www.hindawi.com
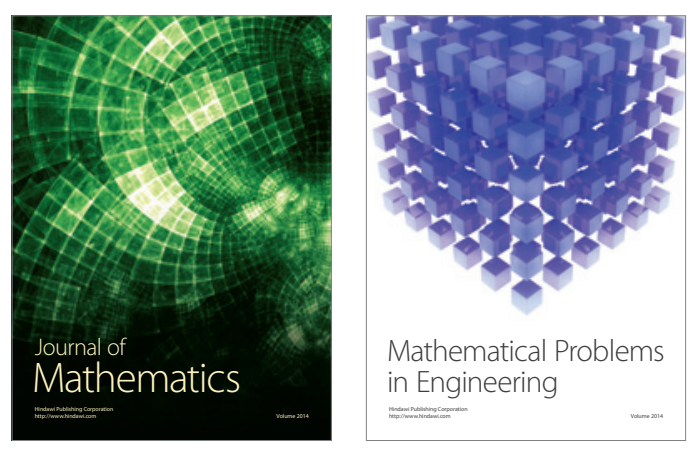

Mathematical Problems in Engineering
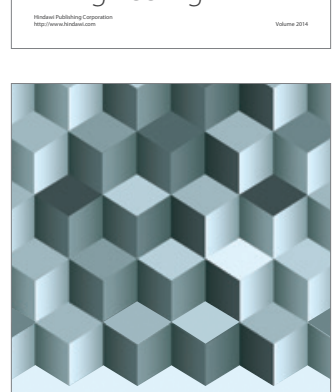

Journal of

Function Spaces
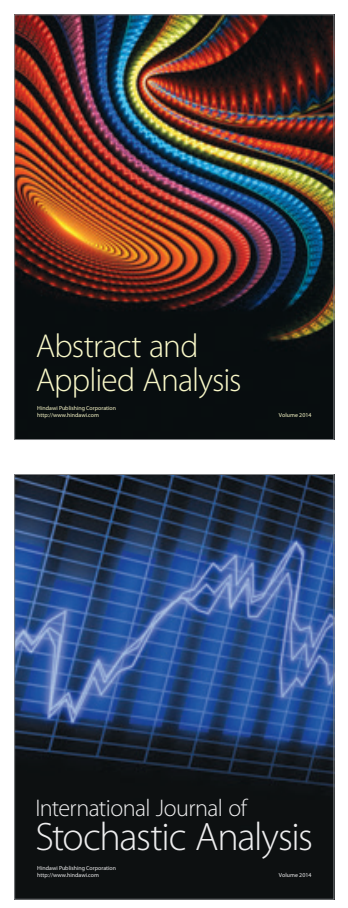

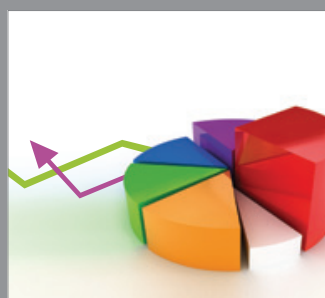

ournal of

Probability and Statistics

Promensencen
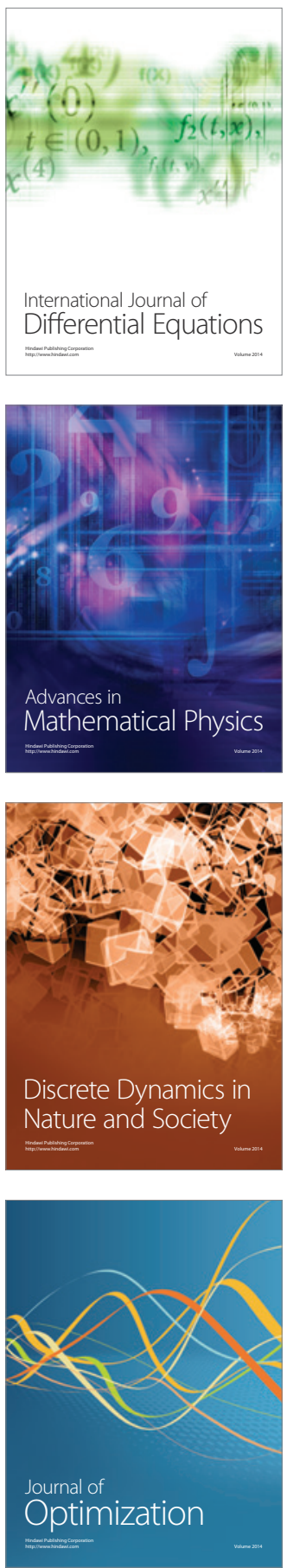